\title{
Marcadores psicológico, fisiológico e bioquímico para determinação dos efeitos da carga de treino e do overtraining
}

\author{
Psychological, physiological and biochemical markers of the \\ training load and the overtraining effects
}

Daniel Schimitz de Freitas

Renato Miranda

Maurício Bara Filho

1 Universidade Federal de Juiz de Fora, Faculdade de Educação Física e Desportos, Juiz de Fora, MG, Brasil.

Recebido em 26/08/08 Revisado em 01/10/08 Aprovado em 13/02/09
Resumo - $\mathrm{O}$ treinamento desportivo é um processo que tem por objetivo romper o equilíbrio interno do organismo humano e assim, aperfeiçoar o desempenho do atleta. No entanto, o estresse provocado pelos treinamentos pode acarretar reações negativas, tal como a síndrome do overtraining. Esta inadaptação pode ser evitada pelo monitoramento dos efeitos do treinamento através de variáveis psicológicas, fisiológicas e bioquímicas. Estudos pesquisados suportam que não há um consenso sobre a eficiência de alguns marcadores, mas são unânimes quanto ao fato de um único marcador não ser capaz de monitorar e prevenir esta síndrome. Fica clara a necessidade de outros estudos para minimizar as dúvidas e aumentar o conhecimento a cerca deste assunto que é de grande interesse dos pesquisadores e profissionais que estão envolvidos com o esporte de rendimento. Mediante isso, o objetivo dessa revisão foi abordar, a partir dos dados da literatura especializada, como os marcadores psicológico, fisiológico e bioquímico, especificamente, estado de humor, escore do questionário de overtraining, variabilidade da frequência cardíaca (VFC) em repouso e creatina quinase plasmática $(\mathrm{CK})$, relacionam-se com a demanda de treinamento e o overtraining.

Palavras-chave: Treinamento; Marcadores; Rendimento.

Abstract - Athletic training is a process aimed at breaking the internal balance of the human organism and thus to improve the athlete's performance. However, training stress may result in negative responses such as overtraining syndrome. This maladaptation can be avoided by monitoring the effects of training using psychological, physiological and biochemical variables. No consensus exists regarding the efficiency of some markers, but studies are unanimous that $s$ single marker is unable to monitor and prevent this syndrome. Further studies are necessary to clarify doubts and to broaden the knowledge about this topic, which is of marked interest to researchers involved in high performance sports. Thus, the purpose of this review of the specialized literature was to discuss how psychological, physiological and biochemical markers, such as mood state, overtraining score, resting heart rate variability and creatine kinase, are associated with training load and overtraining.

Key words: Trainning; Markers; Performance. 


\section{INTRODUÇÃO}

O treinamento desportivo é um processo orientado para a melhoria do desempenho do atleta que tem por objetivo romper o equilíbrio interno do organismo humano por meio de um aumento progressivo das cargas de treinamento ${ }^{1}$. Este processo constitui-se em uma fonte causadora de estresse em consequência de fatores psicofisiológicos que são fundamentais para o rendimento esportivo ${ }^{2}$.

Uma das condições primordiais para um treinamento de qualidade é o contínuo e adequado desequilíbrio da homeostase, caracterizado como estresse positivo. Em sequência, é fundamental que haja um período suficiente de recuperação para a ocorrência da supercompensação ${ }^{3}$. No entanto, o estresse provocado pelos treinamentos pode acarretar reações negativas quando as cargas impostas são incompatíveis com a capacidade de resposta do atleta, que resultará em uma inadaptação psicofísica com possíveis repercussões negativas no rendimento atlético. Essa inadaptação está associada a alterações fisiológicas, bioquímicas e psicológicas, que por sua vez provocará prejuízos para a saúde do atleta, tal como a síndrome do overtraining ${ }^{4-6}$. Em casos extremos, pode evoluir para o fenômeno do burnout, processo no qual há um esgotamento psicofisiológico do atleta sentindo-se este completamente desmotivado com o esporte ${ }^{1,2}$.

A intensidade e o volume do treinamento, assim como o tempo de recuperação entre as sessões sucessivas, têm sido uma grande preocupação para técnicos, preparadores físicos, fisiologistas e cientistas uma vez que estes são fatores intervenientes no treinamento esportivo e há uma linha tênue entre os resultados positivos (aprimoramento da condição física) e negativos (efeitos deletérios) proporcionados pelo estresse das cargas de treino, principalmente, em atletas que estão sempre no limite psicofísico do seu organismo.

\section{OVERREACHING, OVERTRAINING E RECUPERAÇÃO}

O overreaching e o overtraining são processos nos quais o atleta apresenta uma queda no rendimento esportivo, sendo que no primeiro o indivíduo se recupera totalmente em no máximo duas semanas, mas no segundo o período de recuperação pode levar de algumas semanas a meses?

$\mathrm{O}$ overreaching ocorre devido ao acúmulo do estresse do treinamento e de outros fatores que resultam em uma diminuição da capacidade de rendimento, a qual é restaurada de alguns dias a duas semanas ${ }^{2,4,8,9}$. O overreaching pode ainda ser entendido como parte do processo da supercompensação do organismo ${ }^{9}$. Miranda e Bara Filho ${ }^{1}$ propõem uma adaptação do diagrama da Síndrome da Adaptação Geral (SAG) de Selye (Figura 1), com base nos princípios científicos que norteiam o treinamento esportivo, que ilustra perfeitamente este processo.

O overtraining é uma desordem que afeta um grande número de atletas ${ }^{10}$ e pode ser caracterizada como uma síndrome complexa na qual há um desequilíbrio entre o estresse do treinamento bem como de outros fatores e a recuperação $0^{2,8,11,12}$, ou ainda, uma condição na qual o atleta está treinando excessivamente, com uma baixa qualidade de recuperação, sente-se mentalmente fatigado e apresenta uma deterioração do desempenho $\mathrm{o}^{10,13-15}$. De acordo com Varlet-Marie et al. ${ }^{5}$, a síndrome do overtraining pode ser entendida como o terceiro estágio da SAG de Selye que é o esgotamento.

$O$ atleta com a síndrome do overtraining, além da dificuldade em manter os regimes de treinamento com consequente queda no desempenho, pode apresentar infecção do trato respiratório superior, imunossupressão, percepção de pernas pesadas, fadiga generalizada, aumento da percepção subjetiva do esforço, alterações da frequência cardíaca,

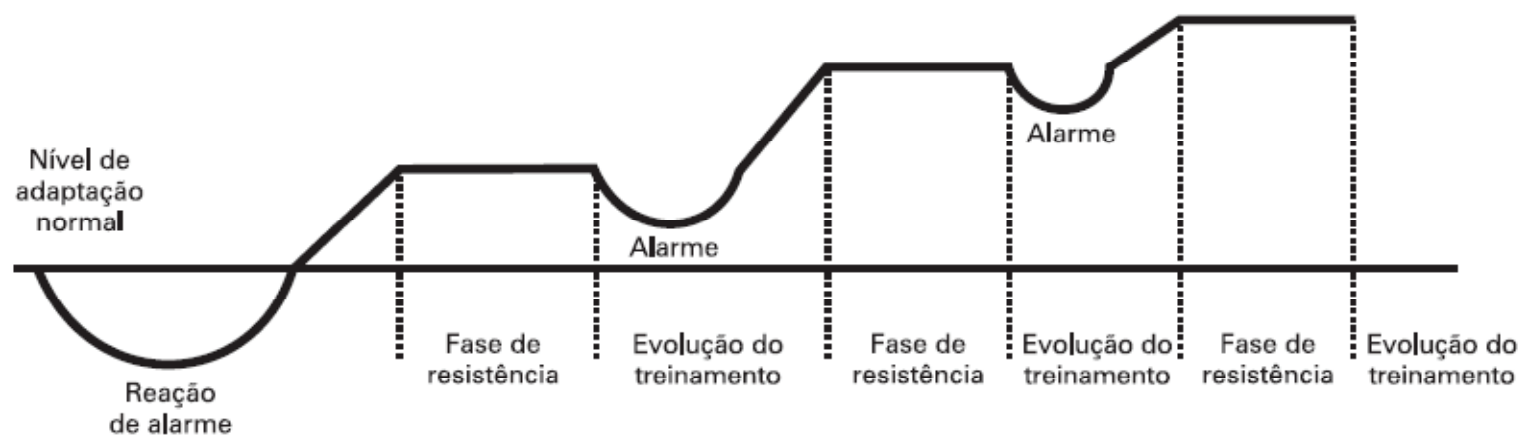

Figura 1. Adaptação do diagrama da SAG para o treinamento esportivo. Fonte: Livro Construindo um atleta vencedor: Uma abordagem psicofísica do esporte, 2008 p. 93. Autorizado pelos autores. 
disfunções no sistema nervoso autônomo, distúrbios do sono e do apetite, alterações de humor, depressão, entre outros ${ }^{2,4-7,72-14}$.

Poucos estudos indicaram uma descrição precisa do processo do overtraining para entender o padrão de adaptações positivas e negativas às cargas de treinamento ${ }^{16}$. Fatores como a monotonia dos treinamentos, o excesso de pressão e de competições podem contribuir para o overtraining, mas o principal fator causal dessa síndrome é a recuperação inadequada ${ }^{2,17}$.

A recuperação pode ser definida como a compensação do estado de déficit de um organismo ou o restabelecimento do estado homeostático ${ }^{17}$. Devido às tensões, preocupações e altas expectativas por sucesso que o cenário do alto nível competitivo reverbera, são observadas falhas na aplicação da metodologia de treinamentos de atletas, principalmente, com relação aos intervalos de recuperação. $\mathrm{O}$ repouso adequado após o exercício intenso pode ser importante para melhorar a recuperação psicológica e fisiológica do atleta $^{18}$. Quanto maior a demanda do treinamento, maior a necessidade de recuperação do atleta ${ }^{2}$.

Quando os períodos de recuperação dos atletas não são adequados e as cargas são aumentadas desproporcionalmente, a rotina do atleta pode se tornar cada vez mais extenuante ${ }^{6}$ e muitas das alterações fisiológicas associadas com o treinamento físico podem ser revertidas ao overtraining ${ }^{1}$.

Diferenças interindividuais no potencial de recuperação, capacidade de realizar o exercício, estressores não relacionados especificamente ao treinamento (família, estudos, trabalho e outros) e a tolerância ao estresse podem explicar porque os atletas apresentam respostas diferentes para as mesmas cargas de treinamento ${ }^{11}$.

Os fatores associados ao overtraining podem prejudicar a saúde do atleta e afastá-lo momentaneamente de treinamentos e competições, podendo comprometer completamente a carreira do mesmo. Admite-se que a principal causa desta síndrome é o tempo inadequado de recuperação. A relação direta entre recuperação e estresse da carga de treino reforça a necessidade da periodização dos programas de treinamento para que os resultados sejam alcançados sem comprometer a saúde e a integridade psicofísica e social do atleta, que é o foco principal do processo.

\section{ESTUDOS COM MARCADORES DA CARGA DE TREINAMENTO}

Apesar dos avanços nas pesquisas com as cargas de treinamento, ainda não foi identificado um marcador confiável, simples e específico para monitorar regularmente a resposta do atleta à carga de treinamento $0^{2,10}$ e para diagnosticar o overreaching e o overtraining nos estágios iniciais ${ }^{7,10,13}$ ,pois nenhum parâmetro isolado é suficiente para avaliá-los e predizê-los ${ }^{14}$. Assim, o monitoramento do treinamento de atletas de elite deveria envolver uma avaliação multivariada para mensurar a adaptação a certas cargas de treino?.

Os sintomas associados ao overtraining podem ser divididos em quatro categorias de avaliação: psicológicos, fisiológicos, bioquímicos e imunológicos ${ }^{2}$ e, de acordo com $\mathrm{Smith}^{4}$, a utilização de marcadores dessas quatro categorias juntos, é a melhor forma de monitorar o treinamento.

Mediante isso, o objetivo dessa revisão é abordar, a partir dos dados da literatura especializada, como os marcadores psicológico, fisiológico e bioquímico, especificamente, estado de humor, escore do questionário de overtraining, variabilidade da frequência cardíaca (VFC) em repouso e creatina quinase plasmática $(\mathrm{CK})$, relacionam-se com a demanda de treinamento e o overtraining.

\section{ESTADO DE HUMOR E O EXERCÍCIO}

Os marcadores psicológicos têm sido largamente utilizados na tentativa de avaliar os efeitos das cargas de treino ${ }^{19}$. Métodos como o Perfil de Estado de Humor (POMS), Questionário de EstresseRecuperação (REST-Q), Escala de Percepção do esforço de Borg (RPE) têm sido utilizados para o acompanhamento e monitoramento dos treinamentos $^{17}$.

As relações entre as dimensões do humor, o rendimento esportivo e a monitorização do treinamento têm sido motivo de preocupações e, consequentemente, de investigações na área da psicologia do esporte e das atividades físicas ${ }^{20}$. Muitos estudos têm avaliado o estado de humor através do POMS - Profile of Mood States ${ }^{21}$ que mede o estresse psicológico através de suas seis escalas - tensão/ ansiedade, depressão, raiva, vigor, fadiga e confusão mental. Desta maneira, este método constitui-se em uma das medidas mais completas para avaliar os efeitos do estresse da carga de treinamentos em variáveis psicológicas.

Grandes aumentos e diminuições no volume e na intensidade do treinamento de resistência têm sido encontrados, estando associados com mudanças nos pontos da fadiga e vigor do POMS em um padrão dose-resposta ${ }^{18}$. Estudos que têm observado a relação entre o exercício e a condição 
psicológica concluíram que o exercício intenso afeta negativamente o estado de humor ${ }^{22}$.

Experimentos com atletas de resistência têm revelado que a atividade física pode tanto melhorar quanto piorar a fadiga e a energia do humor, e o efeito depende do volume e da intensidade de estímulo do treinamento ${ }^{19,20}$. As alterações no estado de humor são relacionadas tanto à prática regular de atividades físicas quanto aos diferentes momentos de treinamento e competições esportivas. No estudo de Halson et al..$^{12}$, foi constatada uma alteração no estado de humor em ciclistas em resposta ao treinamento intenso. Uma relação inversa, mas não estatisticamente significativa, entre o vigor e a fadiga, avaliados pelo POMS, foi observada nos estudos de Baumert et al..$^{8}$ e Silva et al. ${ }^{20} \mathrm{com}$ corredores e triatletas. Buchheit et al..$^{23}$, em seu estudo com indivíduos jovens em diferentes níveis de condicionamento, não encontraram diferença significativa no estado de humor ou no nível de fadiga entre os indivíduos sedentários, moderadamente treinados e altamente treinados.

O estresse provocado pelo treinamento influencia positiva ou negativamente o estado mental do atleta. Algumas variáveis psicológicas são mais responsivas a esse estresse e podem ser avaliadas através de questionários. Esse procedimento, apesar de algumas limitações como a subjetividade das respostas, pode ajudar no controle dos efeitos da carga de treino, otimizando o aprimoramento da condição física e auxiliando na prevenção de efeitos negativos como a síndrome do overtraining.

\section{ESCORE DO QUESTIONÁRIO DE OVERTRAINING E O EXERCÍCIO}

O questionário de overtraining SFMS (Société Française de Médicine du Sport) ${ }^{24}$ foi desenvolvido para quantificar os sintomas clínicos iniciais da síndrome do overtraining ${ }^{25}$,visto que apresenta uma relação direta com cargas elevadas de treinamento ${ }^{5}$. Este instrumento é capaz de medir o estresse psicofisiológico e ajuda a avaliar o grau de sobrecarga em atletas submetidos a programas de treinamento intenso, através de 54 perguntas respondidas como sim ou não, sendo avaliado através do escore de respostas positivas ${ }^{24,25}$. De acordo com Varlet-Marie et al. ${ }^{5}$, este questionário é uma ferramenta importante no diagnóstico da síndrome do overtraining.

Devido a sua utilização em diversos estudos, este questionário já foi traduzido para a língua portuguesa (Brasil) ${ }^{26}$ e está em processo avançado de validação com algumas modificações como a divisão das afirmativas em 6 escalas (rendimento, psicológico, fisiológico, nutricional, social e imunológico) e as respostas pela escala de Likert de 0 a 3 (0-nunca, 1 - às vezes, 2 -frequentemente e 3 - sempre).

O escore do questionário SFMS parece estar correlacionado com marcadores do dano muscular como a creatina quinase (CK) e a miosina ${ }^{5}$ e com a viscosidade plasmática ${ }^{13,25}$ e pode escanear e detectar precocemente a síndrome do overtraining, favorecendo a recuperação do atleta ${ }^{24}$.

Este questionário é uma ferramenta que vem sendo usada há pouco tempo e parece ser capaz de detectar os efeitos negativos do treinamento, podendo ser utilizado para o controle das cargas de treino. Assim, a utilização do questionário de overtraining merece mais estudos, associando-o a outras variáveis e com atletas brasileiros para verificar a sua aplicação nesta população.

\section{VARIABILIDADE DA FREQUÊNCIA CARDÍACA (VFC) E O EXERCÍCIO}

O estresse fisiológico do treinamento é o principal aspecto associado ao overtraining ${ }^{2}$. Nos últimos anos, tem-se observado um aumento no interesse pela forma como o sistema cardiovascular reage ao estresse do exercício ${ }^{27}$ e pelo uso de mensurações não-invasivas para examinar a função autonômica cardíaca (simpática e parassimpática) ${ }^{28}$.

A VFC tem o potencial de avaliar, de forma não-invasiva, a oscilação temporal dos intervalos R-R através de métodos específicos no domínio do tempo e da frequência ${ }^{27,29,30}$. Métodos no domínio do tempo como o SDNN (Desvio padrão de todos os intervalos R-R), RMSSD (Raiz quadrada da média das diferenças sucessivas ao quadrado, entre R-R adjacentes) e o pNN50 (Percentagem das diferenças sucessivas entre os intervalos R-R que são > 50 ms) e no domínio da frequência como o componente HF (Componente espectral de alta frequência $(0,15-0,4 \mathrm{~Hz}))$ apresentam grande correlação com o sistema nervoso parassimpático, sendo, assim, utilizados como marcadores dessa porção do sistema nervoso autônomo. Com relação ao sistema nervoso simpático, ainda não há um consenso sobre alguma variável que possa ser usada para marcar especificamente a sua influência ${ }^{27}$. A avaliação da VFC pode ser feita através da plotagem de Poincaré, na qual cada intervalo R-R é plotado em função do intervalo prévio e permite uma análise visual da atividade do sistema nervoso autônomo, sendo que quando a alça parassimpática é dominante, o escaterograma tem uma forma elíptica e quando 
o simpático é dominante, observa-se uma forma estreita de plotagem ${ }^{31}$. Um exemplo deste modelo pode ser observado na Figura 2, através da qual é possível observar uma diferença entre dois atletas na resposta adaptativa ao treinamento.

A função neurocardíaca pode ser mensurada através da VFC, pois esta variável fisiológica é capaz de quantificar, através de uma estimativa indireta $^{29}$, a interação entre as alças simpática e parassimpática do nodo sinoatrial ${ }^{8,28,31,32}$. Assim, a VFC tem sido um dos parâmetros utilizados para quantificar o estresse gerado pelo exercício, visto que reflete a resposta do sistema nervoso autônomo relativo às alterações de cargas, execuções sucessivas e fadiga ${ }^{8,15,28,29,31}$.

Sabe-se que o treinamento de resistência melhora a função cardiorrespiratória ${ }^{30}$ e aumenta significativamente a VFC tanto no domínio do tempo quanto no domínio da frequência por aumentar a atividade vagal ${ }^{32}$. Este aumento do tônus parassimpático está associado aos efeitos cardioprotetores proporcionados pelo exercício comumente observado em atletas e indivíduos treinados ${ }^{31}$. Mas, no estudo de caso realizado por Hedelin et al. ${ }^{33}$, foi sugerido que o aumento da atividade parassimpática está relacionado ao overtraining.
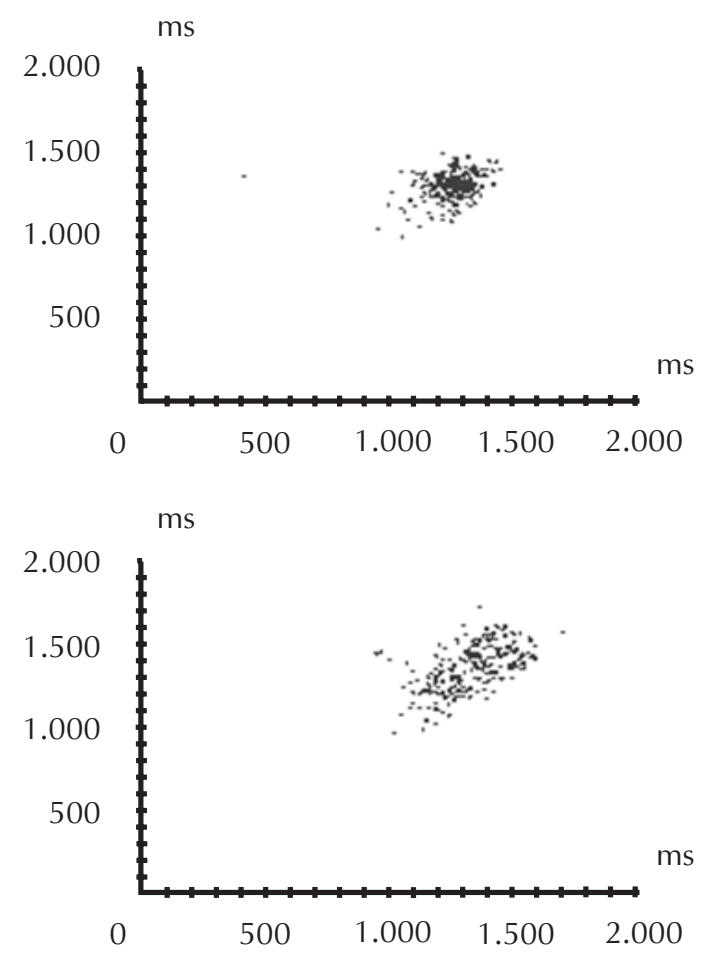

Atleta 1
Uma quantidade moderada de exercício é suficiente para aumentar o índice vagal relacionado à VFC ${ }^{23}$. Mas, de acordo com Mourot et al..$^{34}$, um programa de treinamento intenso ou suficientemente longo parece ser necessário para induzir mudanças significativas na VFC, no entanto, a característica do treinamento (constante, intermitente ou intervalado e a intensidade, duração e frequência das sessões de treino) que provavelmente causam estas mudanças, permanece obscura. Se o exercício intenso for acompanhado de uma recuperação insuficiente, o balaço autonômico pode ser alterado, apresentando uma diminuição do tônus parassimpático e principalmente uma elevação do tônus simpático, o que, associado a outros parâmetros, pode caracterizar o overtraining $^{29}$. Baumert et al. ${ }^{8}$ avaliaram o impacto do aumento da carga de treinamento sobre o sistema cardiovascular em atletas, e constataram que quando o treinamento físico é intensificado abruptamente, a atividade cardiovascular é alterada com a diminuição da atividade parassimpática e elevação da atividade simpática e que essa resposta pode ser monitorada pela VFC em repouso.

Earnest et al. ${ }^{29}$ examinaram a relação entre a VFC e o exercício físico em ciclistas profissionais e concluíram que as alterações na VFC em repouso
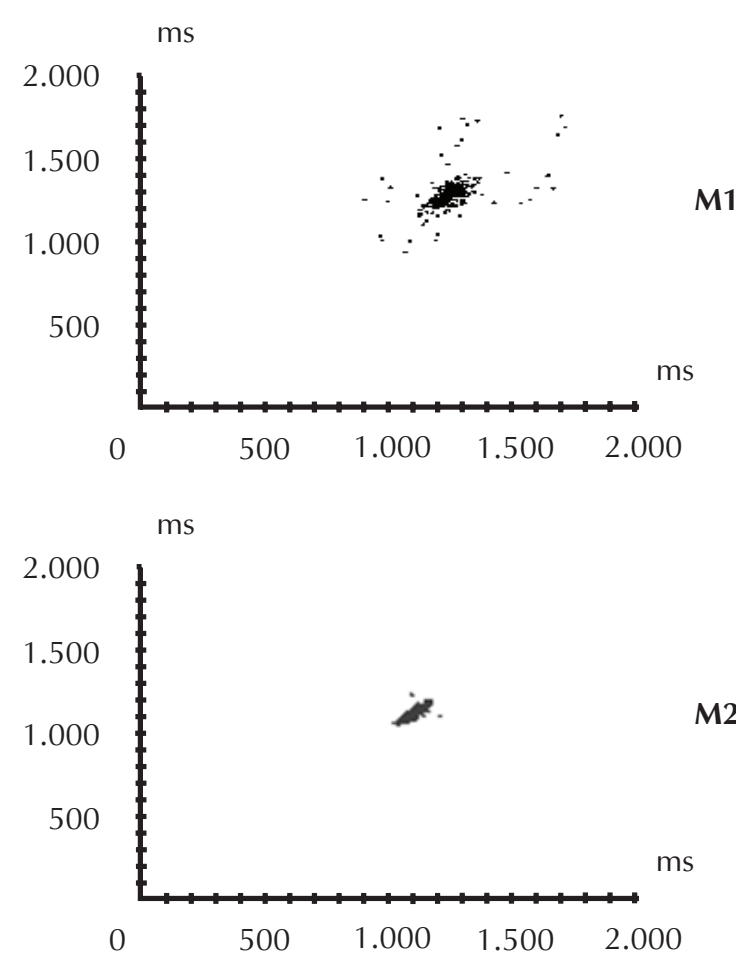

Atleta 2

Figura 2. Plotagem de Poincaré da Variabilidade da Frequência Cardíaca em repouso de dois atletas profissionais de futebol submetidos ao mesmo programa de treinamento. M1 é a avaliação pré e M2 é referente ao momento final após 14 dias de treino. (Bara Filho et al.; dados não publicados). 
são inversamente relacionadas ao volume e à intensidade do exercício e se devem ao esforço cumulativo dos estágios consecutivos da competição. No mesmo estudo, foi observado que, para um esforço semelhante, há uma variação na resposta autonômica cardíaca entre os indivíduos. No estudo de Du et al. ${ }^{30}$ com maratonistas do sexo feminino, foi constatado que maiores níveis de VFC em repouso estavam associados com uma rápida recuperação da frequência cardíaca após o exercício e que isto estaria relacionado a um melhor condicionamento físico. Mourot et al. ${ }^{31}$, em seu estudo com atletas, observaram que o padrão de resposta da VFC em repouso de um atleta em overtraining é semelhante ao de uma indivíduo sedentário e que um atleta, em condições normais, apresenta maior VFC em relação a outros indivíduos menos treinados devido a um maior predomínio vagal. Os autores concluíram, ainda, que a diferenciação entre um indivíduo bem treinado e um em overtraining pode ser feita através da plotagem de Poincaré. Mas, no estudo de Buchheit et al. ${ }^{23}$, foi observado um padrão semelhante do índice vagal da VFC entre sedentários e indivíduos altamente treinados. Pode-se levantar a hipótese de que os indivíduos altamente treinados deste estudo estavam sofrendo da síndrome do overtraining.

O distúrbio do sistema nervoso autônomo tem sido sugerido como um dos sintomas associados ao overtraining $^{33}$. A VFC pode ser usada no controle da carga de treino, principalmente, quando analisa os atletas individualmente ${ }^{3}$, e parece ser um marcador adequado para o overreaching ${ }^{8}$. Esse fato fortalece a importância da avaliação deste parâmetro fisiológico no acompanhamento dos treinamentos de atletas, facilitando o entendimento de como o organismo do indivíduo responde às cargas impostas. A partir das conclusões a respeito da VFC, pode-se ter uma ideia do nível de adaptação do atleta e, se necessário, alterar a periodização. Deve-se ressaltar que esta é uma técnica não-invasiva o que facilita a sua utilização no dia-a-dia dos treinamentos. Mas, ainda há controvérsias sobre as condições de aquisição e análise dos dados, tipo de esporte e treinamento e sobre os resultados obtidos, o que exalta a necessidade de novos estudos. Associado a isso, este método de controle ainda não está ao alcance de grande parte dos preparadores físicos devido ao desconhecimento do modelo e, talvez, ao custo do material.

\section{CREATINA QUINASE (CK) E O EXERCÍCIO}

Marcadores bioquímicos podem ser considerados potentes parâmetros na avaliação da ocorrência do overtraining ${ }^{16}$. Esta síndrome é acompanhada por uma resposta significativa de biomarcadores do estresse oxidativo, os quais são alterados durante períodos de treinamento intenso e retornam aos níveis normais quando a carga diminui, indicando uma relação dose-resposta ${ }^{10}$. Muitos métodos diretos e indiretos têm sido utilizados na análise do dano muscular decorrente do exercício físico ${ }^{35}$. Os métodos indiretos como mioglobina, lactato desidrogenase (LDH), fragmentos da cadeia pesada da miosina e CK, são mais frequentemente usados ${ }^{6}$. Estas moléculas podem ser utilizadas como marcadores do dano no tecido muscular esquelético devido ao fato de serem citoplasmáticas e assim, impermeáveis na membrana plasmática ${ }^{35}$. Dessa forma, o aumento nos níveis dessas moléculas no líquido extracelular pode indicar uma alteração da permeabilidade da membrana ou o rompimento da mesma ${ }^{36}$.

A resposta de marcadores bioquímicos como a $\mathrm{CK}$, em relação às cargas de treinamento tem sido o foco de vários estudos ${ }^{9,37}$. A CK tem um papel chave na formação de energia nas células musculares, visto que é a enzima intramuscular responsável por manter níveis adequados de ATP durante a contração muscular ${ }^{38}$. Algumas hipóteses como hipóxia tecidual, depleção do glicogênio muscular, peroxidação lipídica e acúmulo de espécies reativas de oxigênio (ERO's), são levantadas para justificar a elevação dos níveis de $\mathrm{CK}^{35}$.

Sabe-se que o uso desse marcador para o controle das cargas de treinamento e o diagnóstico do overtraining ainda está em discussão ${ }^{11,14}$ e que as alterações na atividade da $\mathrm{CK}$, após o exercício, variam com as diferentes condições de exercício ${ }^{36}$. Aliado a isso, a avaliação clínica do dano muscular induzido pelo exercício é muito difícil ${ }^{39}$. Mas a CK pode ser utilizada como marcador de fadiga e sobrecarga em indivíduos não-atletas ${ }^{36}$. De acordo com Katirji \& Jaberi ${ }^{38}$ e Gleeson ${ }^{7}$, o exercício físico prolongado e extenuante aumenta os níveis de CK, sendo que a magnitude dessa elevação está diretamente relacionada à intensidade e duração da atividade. Acredita-se que o efeito de sessões sucessivas de exercício prolongado possa desencadear mecanismos que induzam o extravasamento de CK na corrente sanguínea ${ }^{13}$. Segundo Hartmann \& Mester ${ }^{14}$ a determinação da CK parece ser o parâmetro sensível e confiável para avaliar qualquer aumento no estresse muscular ou a tolerância individual ao exercício muscular. Valores de CK total acima de 500UI/L têm sido utilizados como parâmetro para indicar lesão ao tecido muscular $^{39}$. Totsuka et al. ${ }^{36}$ adotaram um valor de 300 
a 500UI/L para indicar que o limite da habilidade muscular havia sido excedido e nomearam-no de "break point" da CK. Deve-se destacar que valores da ordem de 200 a $250 \mathrm{UI} / \mathrm{L}$ podem ser considerados normais para homens atletas ${ }^{14}$.

Purge et al. ${ }^{9}$, em seu estudo, examinaram as alterações da atividade da CK em remadores masculinos de elite durante um período de preparação e constataram que essas mudanças têm um padrão similar às alterações no volume semanal de treinamento. Martínez-Amat et al..$^{39}$ observaram uma diferença na atividade da CK total entre indivíduos saudáveis e aqueles com danos no tecido muscular. Um aumento nos níveis de CK plasmático correspondente à intensificação do treinamento, também foi observado no estudo de Halson et al. ${ }^{12}$ quando estudaram a resposta dessa variável ao overreaching em oito ciclistas durante um período de seis semanas. Hartmann Mester ${ }^{14}$ observaram um aumento considerável na $\mathrm{CK}$, em um grande número de atletas, após treinamento de resistência e de força de intensidade de moderado a intenso. Já no estudo de Zoppi et al. ${ }^{37}$, não foi observada nenhuma diferença significativa na atividade da CK plasmática nos atletas durante uma temporada competitiva no futebol. Ainda nesse estudo, observou-se que as concentrações plasmáticas da CK estiveram sempre acima dos valores de referência para sujeitos sedentários.

De acordo com Hartmann e Mester ${ }^{14}$, há uma clara necessidade de determinações regulares de CK dentro de um programa de mensurações para acompanhar o treinamento. A mensuração da CK plasmática pode ser importante na quantificação do efeito da carga de trabalho durante um macrociclo de treinamento para adequar o intervalo de recuperação devido a sua relação direta com o estresse tecidual. Apesar de ser um método indireto e invasivo, quando comparado a métodos diretos de mensuração do dano muscular como as análises de amostras de músculo ou de imagem por técnica de ressonância magnética, este se mostra como um parâmetro mais acessível e com menor custo para os profissionais que estão envolvidos no processo de treinamento.

\section{RELAÇÃO ENTRE AS VARIÁVEIS}

A determinação de variáveis psicológicas, fisiológicas e bioquímicas, em relação ao exercício, tem sido realizada devido à pertinente relação existente entre as mesmas ${ }^{2}$.

Vários estudos detectaram uma relação direta do estado de humor com a variabilidade da frequência cardíaca (VFC) ${ }^{22,23}$, níveis plasmáticos de leucócitos e razão glutamato/glutamina ${ }^{12} \mathrm{e}$ indireta com a creatina quinase $(\mathrm{CK})$ e lactato desidrogenase ${ }^{18}$ em relação ao exercício. O escore do questionário SFMS de overtraining apresentou relação direta com a carga de treino $\mathrm{o}^{24}$ e com outros marcadores como a $\mathrm{CK}^{5}$, hematócrito e viscosidade plasmáti$\mathrm{ca}^{13,25}$ e indireta com a ferritina ${ }^{5,25}$. A VFC foi relacionada a outros marcadores como a concentração de hemoglobina e células sanguíneas vermelhas em canoístas para identificar o overreaching ${ }^{15}$, mas não foi observada uma relação significativa entre as mesmas. Hedelin et al..$^{33}$ observaram relação direta entre a queda no rendimento e o hormônio luteinizante em um atleta com a síndrome do overtraining, mas nenhuma relação siginificativa com os leucócitos totais, hemoglobina, cortisol e ferritina. Neste estudo, foi observada uma relação indireta entre o rendimento e a VFC.

No entanto, observa-se uma carência de estudos que analisaram, na mesma pesquisa, a correlação entre os marcadores psicológicos, bioquímicos e fisiológicos em relação às cargas de treinamento. A combinação dessas variáveis tem sido a melhor estratégia para identificar o efeito das cargas de treinamento sobre o atleta? ${ }^{7}$. Essa estratégia de monitoramento se apresenta com grande relevância, no meio esportivo, devido à sua importância tanto para se atingir um melhor desempenho quanto para prevenir possíveis excessos que poderiam acarretar o overtraining.

\section{CONSIDERAÇÕES FINAIS}

A utilização de marcadores fisiológicos, psicológicos e bioquímicos na prevenção dos efeitos negativos das cargas de treinamento ainda não está clara. Assim, é notória a necessidade do desenvolvimento de um método acessível e fácil para monitorar o treinamento ${ }^{2,5}$. Entende-se que um marcador ideal do início do overtraining deveria ser sensível às cargas de treinamento, não ser afetado por outros fatores, ser relativamente fácil de mensurar e não muito caro. Atletas de elite e seus técnicos precisam de um sistema para monitorar o treinamento e a recuperação com marcadores confiáveis para serem utilizados na prevenção do overtraining. Kellmann ${ }^{17}$ afirma que há consideráveis vantagens em prevenir o overtraining ao invés de simplesmente preocupar-se com o tratamento e o repouso total.

Apesar disso, técnicas confráveis para a detecção do início do overtraining ainda não foram estabelecidas e, devido a um grande número de 
sintomas e à ausência de um aspecto uniforme desta síndrome, um diagnóstico é difícil ${ }^{34}$. Enquanto nenhum marcador puder ser tomado como um indicador do overtraining precocemente, o monitoramento regular do rendimento e de variáveis fisiológicas, psicológicas e bioquímicas poderia fazer parte da estratégia do treinamento.

Assim, entende-se que estudos com marcadores das três categorias abordadas deveriam ser desenvolvidos, correlacionando-os entre si e com as alterações do volume e intensidade do treinamento. Essa correlação poderia ser estabelecida com os diferentes intervalos e formas para recuperação. A avaliação do técnico e do atleta quanto ao rendimento do mesmo em relação a esses marcadores também seria de grande importância para o meio esportivo competitivo. Acredita-se que outras pesquisas precisam ser realizadas com o monitoramento regular, talvez semanal, das variáveis discutidas nessa revisão, durante um macrociclo de treinamento. Dessa forma, a carga de treino pode ser melhor dimensionada, evitando uma resposta negativa do atleta e minimizando o risco do mesmo vir a desenvolver a síndrome do overtraining.

\section{REFERÊNCIAS BIBLIOGRÁFICAS}

1. Miranda R, Bara Filho M. Construindo um atleta vencedor: Uma abordagem psicofísica do esporte. Porto Alegre: Artmed; 2008.

2. Kenttä G, Hassmén P. Overtraining and recovery. Sports Med 1998;26(1):1-16.

3. Garet M, Tournaire N, Roche F, Laurent R, Lacour JR, Barthélemy JC, et al. Individual interdependence between nocturnal ASN activity and performance in swimmers. Med Sci Sports Exerc 2004;36(12):2112-2118.

4. Smith LL. Overtraining, excessive exercise, and altered immunity. Sports Med 2003;33(5):347-364.

5. Varlet-Marie E, Maso F, Lac G, Brun J-K. Hemorheological disturbances in the overtraining syndrome. Clin Hemorheol Microcirc 2004;30(3-4):211-218.

6. Rogero MM, Mendes RR, Tirapegui J. Aspectos neuroendócrinos e nutricionais em atletas com overtraining. Arq Bras Endocrinol Metab 2005;49(3):359-368.

7. Gleeson M. Biochemical and immunological markers of overtraining. J Sports Sci Med 2002;1(2):31-41.

8. Baumert M, Brechtel L, Lock J, Hermsdorf M, Wolff $\mathrm{R}$, Baier V, et al. Heart rate variability, blood pressure variability, and baroreflex sensitivity in overtrained athletes. Clin J Sports Med 2006;16(5):412-417.

9. Purge P, Jürimäe J, Jürimäe T. Hormonal and psychological adaptation in elite male rowers during prolonged training. J Sports Sci 2006;24(10):1075-1082.

10. Margonis K, Fatouros IG, Jamurtas AZ, Nikolaidis MG, Douroudos I, Chatzinikolaou A, et al. Oxidative stress biomarkers responses to physical overtraining: Implications for diagnosis. Free Radic Biol Med 2007;43(6):901-910.
11. Lehmann C, Foster C, Keul J. Overtraining in endurance athletes: a brief review. Med Sci Sports Exerc 1993;25(7):854-862.

12. Halson SL, Lancaster GI, Jeukendrup AE, Gleeson M. Immunological responses to overreaching in cyclists. Med Sci Sports Exerc 2003;35(5):854-861.

13. Varlet-Marie E, Gaudard A, Mercier J, Bressolle F, Brun J-F. In the feeling of heavy legs in overtrained athletes related to impaired hemorheology?. Clin Hemorheol Microcirc 2003;28(3):151-159.

14. Hartmann U, Mester J. Training and overtraining markers in selected sport events. Med Sci Sports Exerc 2000;32(1):209-215.

15. Hedelin R, Wiklund U, Bjerle P, Henriksson-Larsén K. Cardiac autonomic imbalance in an overtrained athlete. Med Sci Sports Exerc 2000;32(9):1531-1533.

16. Petibois C, Cazorla G, Déleris G. The biological and metabolic adaptations to 12 months training in elite rowers. Int J Sports Med 2003;24(1):36-42.

17. Kellmann M. Enhancing recovery: Preventing underperformance in athletes. Champaign, IL: Human Kinetics; 2002.p.3-24.

18. Suzuki M, Umeda T, Nakaji S, Shimoyama T, Mashiko $\mathrm{T}$, Sugawara K. Effect of incorporating low intensity exercise into the recovery period after a rugby match. Br J Sports Med 2004;38(4):436-440.

19. O'connor PJ, Puetz TW. Chonic physical activity and feelings of energy and fatigue. Med Sci Sports Exerc 2005;37(2):299-305.

20. Silva MV, Freitas DS, Castro PL, Lima JP, Bara Filho M. Análise do efeito da carga de treinamento sobre os sentimentos de vigor e fadiga durante um macrociclo de treinamento. Coleção Pesquisa em Educação Física 2007;6(1):73-78.

21. McNair DM, Lorr M, Droppleman LF. Profile of Mood States Manual. San Diego: Educational and Industrial Testing Service; 1971.

22. Sakuragi S, Sugiyama Y. Effects of daily walking subjective symptoms, mood and autonomic nervous function. J Physiol Anthropol 2006;25(4):281-289.

23. Buchheit M, Simon C, Piquard F, Ehrhart J, Brandenberger G. Effects of increased training load on vagal-related indexes of heart rate variability: a novel sleep approach. Am J Physiol Heart Circ Physiol 2004;287(6):H2813-H2818.

24. Brun J-F. The overtraining: To a system of evaluation usable by routine examination. Sci Sports 2003;18(6):282-286.

25. Gaudard A, Varlet-Marie E, Bressolle J, Mercier L, Brun J-F. Hemorheological correlates of fitness and unfitness in athletes: moving beyond the apparent "paradox of hematocrit"?. Clin Hemorheol Microcirc 2003;28(3):161-173.

26. Fernandes J, Nogueira R, Andrade F, Freitas DS, Bara Filho M. Tradução e adaptação do questionário de sintomas clínicos do overtraining. Coleção Pesquisa em Educação Física 2008;7(1):335-340.

27. Task Force of the European Society of Cardiology and the North American Society of Pacing and Electrophysiology. Heart rate variability: standards of measurement, physiological interpretation and clinical use. Eur Heart J 1996;17(3):354-381. 
28. Pober DM, Braun B, Freedson PS. Effects of a single bout of exercise on resting heart rate variability. Med Sci Sports Exerc 2004;36(7):1140-1148.

29. Earnest CP, Jurca R, Church TS, Chicharro JL, Hoyos J, Lucia A. Relation between physical exertion and heart rate variability characteristics in professional cyclists during the Tour of Spain. Br J Sports Med 2004;38(5):568-575.

30. Du N, Bai S, Oguri K, Kato Y, Matsumoto I, Kawase H, et al. Heart rate recovery after exercise and neural regulation of heart rate variability in 30-40 year old female marathon runners. J Sports Sci Med 2005;4(1):9-17.

31. Mourot L, Bouhaddi M, Perrey S, Cappelle S, Henriet M-T, Wolf J-P, et al. Decrease in heart rate variability with overtraining: assessment by the Poincaré plot analysis. Clin Physiol Funct Imaging 2004;24(1):10-18.

32. Madden KM, Levy WC, Stratton JR. Exercise training and heart rate variability in older adult female subjects. Clin Invest Med 2006;29(1):20-28.

33. Hedelin R, Kenttá G, Wiklund U, Bjerle P, HenrikssonLarsén K. Short-term overtraining: effects on performance, circulatory responses, and heart rate variability. Med Sci Sports Exerc 2000;32(8):1480-1484.

34. Mourot L, Tordi N, Perrey S, Bouhaddi M, Rouillon J-D, Regnard J. Overall increase in heart rate variability after the Square-Wave Endurance Exercise Test training. Sci Sports 2005;20(2):83-90.

35. Foschini D, Prestes J, Charro MA. Relação entre exercício físico, dano muscular e dor muscular de início tardio. Rev Bras Cineantropom Desempenho Humano 2007;9(1):101-106.
36. Totsuka M, Nakaji S, Suzuki K. Break point of serum creatine kinase release after endurance exercise. J Appl Physiol 2002;93(4):1280-1286.

37. Zoppi CC, Antunes-Neto J, Catanho FO, Goulart LF, Motta e Moura N, Macedo DV. Alterações em biomarcadores de estresse oxidativo, defesa antioxidante e lesão muscular em jogadores de futebol durante uma temporada competitiva, SC. Rev Paul Educ Fís 2003;17(2):119-130.

38. Katirji B, Al-Jaberi MM. Creatine kinase revisited. J Clin Neuromusc Dis 2001;2(3):158-163.

39. Martínez-Amat A, Boulaiz H, Prados J, Marchal JÁ, Puche PP, Caba O, et al. Release of a-actin into serum after skeletal muscle damage. Br J Sports Med 2005;39(11):830-834.

\section{Endereço para correspondência}

Daniel Gustavo Schimitz de Freitas

Rua Itamar Soares de Oliveira, 47 / 202

Bairro Cascatinha

36033280 - Juiz de fora, MG. Brasil

E-mail: danielschimitz@yahoo.com 